\title{
Bullying: Detección mediante el test sociométrico y prevención a través de experiencias basadas en el método socioafectivo
}

\author{
Bullying: Detection Through the Sociometric Test and Prevention Through the Experiences \\ Based on the Socioaffective Method
}

\section{Bullying: Detecção através do teste sociométrico e prevenção por meio de experiências baseadas no método sócioafetivo}

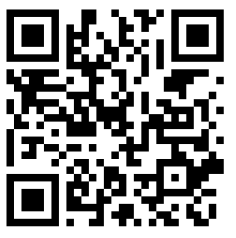

Carolina Hamodi-Galán

Universidad de Valladolid

Soria, España

carolina.hamodi@uva.es

http://orcid.org/0000-0002-0965-8410

Yara de Benito-Brunet

Universidad de Valladolid

Soria, España

yaradb95@gmail.com

https://orcid.org/0000-0003-4736-4090

Recibido • Received • Recebido: 03 / 11 / 2017

Corregido • Revised • Revisado: 28 / 11 / 2018

Aceptado • Accepted • Aprovado: 05/ 06 / 2019

Resumen: El bullying es una de las formas de violencia más comunes en la actualidad escolar. El artículo presenta una experiencia en la que se utiliza la sociometría como metodología para conocer las relaciones existentes en el aula (mediante test sociométrico y matriz sociométrica), junto con otros instrumentos de corte cualitativo (diario de campo y guía de observación) para desarrollar una metodología mixta (cuantitativa y cualitativa). Tras los resultados se plantea una propuesta de intervención para la prevención del bullying que se sustenta sobre el método socioafectivo (método para trabajar empatía). Los resultados obtenidos manifiestan la importancia de desarrollar dinámicas en el aula que trabajen la empatía y habilidades sociales y el impacto positivo que ello supone en el aula.

Palabras claves: Bullying; empatía; método socioafectivo; prevención; test sociométrico.

Abstract: Nowadays, bullying is one of the most common forms of violence at school. This article includes an experience in which sociometry is used as a methodology implemented to know the current relationships between students in the class (through a sociometric test and a sociometric matrix), along with qualitative research tools (field diary and observation guide), in order to develop a mixed methodology (a quantitative and qualitative research design). The results obtained outline the importance of implementing, in class, dynamics that work on empathy and social abilities and the resulting positive impact on the class.

Keywords: Bullying; empathy; socio-affective method; prevention; sociometric test. 
doi: http://dx.doi.org/10.15359/ree.23-3.3

URL: http://www.una.ac.cr/educare

CORREO: educare@una.cr

\begin{abstract}
Resumo: Atualmente, o bullying é uma das formas mais comuns de violência escolar. $\mathrm{O}$ artigo apresenta uma experiência na qual utiliza a sociometria como uma metodologia para conhecer as relações existentes em sala de aula (através de testes sociométricos e matriz sociométrica), juntamente com outras ferramentas qualitativas (diário de campo e guia de observação) para desenvolver metodologia mista (quantitativa e qualitativa). Após os resultados, deriva uma proposta de intervenção para a prevenção do bullying que se baseia no método socio afetivo (método para trabalhar a empatia). Os resultados mostram a importância de desenvolver dinâmicas de sala de aula que trabalhem com empatia e habilidades sociais e o impacto positivo que consequentemente existirá nesse espaço.
\end{abstract}

Palavras chaves: bullying; empatia, método sócioafetivo; prevenção; teste sociométrico

\title{
Introducción
}

El acoso escolar o bullying es uno de los principales problemas al que nos enfrentamos en la actualidad siendo la forma de violencia más común entre niños y jóvenes que encontramos en el contexto escolar. En ocasiones, es observable como dentro de un grupo, uno o algunos miembros ejercen un abuso de poder sobre una víctima, de tal forma, que se acaba convirtiendo en un blanco fácil para los agresores. Este fenómeno no sólo afecta a los acosados, sino también al resto de alumnos, a toda la comunidad educativa y a la sociedad en general. Pero especialmente, ejerce un daño y perjuicio constante sobre quien lo sufre, afectando a su entorno social y personal hasta el punto de llegar a quitarse la vida, tal y como lo reflejan los medios de comunicación. En una de las investigaciones mas recientes sobre este tema (Morales-Ramírez y Villalobos-Cordero, 2017, p. 18) se plantea como necesidad "realizar un diagnóstico en el centro educativo que determine la prevalencia o no del acoso escolar".

Por ello, el principal objetivo planteado en este artículo es doble: a) plantear la sociometría como una rama de la sociología capaz de ayudar a detectar posibles casos de bullying; presentar el método socioafectivo como una metodología muy potente para trabajar en el aula valores como la empatía. Finalmente se presenta una Propuesta de Intervención para la Prevención del Bullying basada en la citada metodología. Como objetivo final se pretende potenciar y mejorar el clima y las relaciones del estudiantado, así como paliar posibles casos de acoso escolar.

\section{Fundamentación teórica}

El bullying es un fenómeno que ha existido siempre y ha sido estudiado a lo largo de los años, pero es en las últimas décadas cuando podemos observar que nuestra sociedad en general, y las comunidades educativas en particular, están manifestando una gran sensibilización hacia este problema (Montañés, Bartolomé, Parra y Montañés, 2009). Los primeros estudios sobre bullying se iniciaron en 1973, y fueron realizados por Olweus (1994), quien entiende este concepto 
como una conducta de persecución física o psicológica que realiza un alumno o alumna contra otra persona a la que elige como víctima de repetidos ataques. Esta acción negativa e intencionada sitúa a la víctima en posiciones de las que difícilmente puede salir por sus propios medios.

Esta definición fue compartida por Farrington (1993), cuya teoría sobre el bullying encierra "un desequilibrio de poder entre el agresor y la víctima que tiene lugar repetidas veces". De esta forma, el bullying adopta la forma de una interacción constante entre el sujeto agresor y la víctima. Coincidiendo con este planteamiento, Palomero y Fernández (2001, p. 26) definen el maltrato entre iguales como "una conducta de persecución y agresión física y psicológica o moral, que realiza un alumno ... sobre otro, con desequilibro de poder y de manera reiterada". Se observa que estos autores ponen el énfasis en la agresión física y el desequilibrio de poder, no obstante "es importante insistir en la necesidad de acotar y definir con claridad el término bullying y evitar utilizarlo como sinónimo de todo tipo de violencia que se presenta en la escuela" (Gómez, 2013, p. 863).

\section{Qué es y qué no es bullying}

Bullying es una palabra de origen anglosajón cuya raíz "bully" significa matón, acosador, abusón. Actualmente, utilizamos este anglicismo para identificar "las reiteradas acciones negativas dirigidas a un niño concreto por parte de uno o más estudiantes" y se entiende este concepto como dependiente de los contextos sociales en los que se produce (Ortega, 2010, p. 81). Muchos han sido los debates e investigaciones que se han llevado a cabo para concluir una definición apropiada para este concepto. Actualmente, sigue sin haber un acuerdo sobre una descripción universal del bullying, no obstante, se percibe cierto consenso acerca de su comportamiento agresivo, que satisface tres criterios (Smith, 2016): (1) repetición, ocurre más de una vez, (2) existe un abuso sistemático de poder, y (3) intencionalidad, entendida como el deseo de herir a propósito a otro sujeto (Olweus, 2013).

Tras la recopilación de distintas definiciones del término bullying (Avilés, 2006; Cerezo, 1997; Farrington, 1993; Gómez, 2013; Olweus, 1994; Ortega, 2010; Palomeroy Fernández, 2001;Smith, 2016) podemos concluir que se trata de una forma ilegítima de confrontación de intereses o necesidades, se desarrolla en la escuela y es llevada a cabo por un grupo o un individuo de forma mantenida en el tiempo en la que existe un desequilibrio de poder entre el sujeto agresor y su víctima.

Pero "no todas las conductas que pueden describirse bajo esta dominación son de igual gravedad e importancia en cuanto a los daños que producen" (Ortega, 2010, p. 17). Existen situaciones escolares en las que se producen malos entendidos entre estudiantes que en realidad no son fenómenos bullying, sino pequeños conflictos que se provocan entre iguales, ya que "el conflicto es, en sí mismo, una fuente de matices que alimenta permanentemente la interacción de los que conviven compartiendo espacios, tiempos, actividades y normas" (Ortega, 2010, p. 17). 
doi: http://dx.doi.org/10.15359/ree.23-3.3

URL: http://www.una.ac.cr/educare

CORREO: educare@una.cr

Coincidiendo con el planteamiento de Montañés etal.(2009,p.2) deque"no hay que confundir el bullying con situaciones escolares de interacción brusca y con situaciones perturbadoras de la convivencia (peleas, bromas, riñas esporádicas, vandalismo escolar, enfrentamientos entre bandas, [etc.])". Estas formas de violencia se parecen al bullying porque conllevan abuso, pretensión, alevosía y perversión social. A pesar de ello, "no es considerado como acoso escolar, ya que no tiene porqué existir un vínculo relacional estable entre las partes, una focalización en los ataques o el desequilibro en el uso y abuso de poder"(Avilés, 2006, p. 83). Olweus (1994) ejemplifica esta idea al indicar que el término bullying no debe ser utilizado en aquellos casos en los que dos personas (o grupos de personas) se enfrentan mediante relaciones de poder iguales (físicas o psicológicas). Por lo tanto, cuando se da violencia escolar no siempre se trata de casos de bullying, ya que no es su única expresión (Echeverri, Gutiérrez, Ramírez y Morales, 2014).

\section{Prevención de bullying: Programas y experiencias}

En el estudio empírico llevado a cabo por Rigby y Smith (2011) sobre la posibilidad del aumento de casos de bullying en 27 países de Europa, Estados Unidos y Australia, los resultados mostraron cambios evidentes entre 1990 y 2008. Se puede observar cómo el acoso escolar ha ido ganando cada vez más terreno en el ámbito educativo. No obstante, destaca el caso de Noruega, donde se manifiesta una notable disminución de casos de acoso escolar a partir de 2004, cuando se iniciaron programas anti-bullying.

La intervención para la prevención del bullying ha sido gradual. En un principio, se llevaron a cabo programas pioneros como el "Olweus Bullying Prevention Program" (1983), basado en un cuestionario individual en el que el propio alumnado podía contar su experiencia en relación con el acoso escolar. De esta forma, algunos casos de bullying podrían ser detectados y, de la misma manera, mitigados.

Uno de los programas de prevención, intervención y seguimiento más conocidos es el llamado KiVa, acrónimo de Kiusaamista Vastaan (contra el acoso escolar), iniciado en Finlandia en 2007. Este método incluye una programación curricular destinada a la prevención de casos de bullying en las aulas, así como la actuación sobre los sujetos espectadores (Williford et al., 2012). Se trata de un método de gran reconocimiento, ya que sus resultados (Williford et al., 2012) muestran evidencias significativas en la reducción de casos de acoso escolar en aquellos países donde se ha implantado el programa.

En cuanto a España, destaca el "programa 'Dando Pasos Hacia la Paz' que tiene como finalidad fomentar la convivencia, el respeto por los derechos humanos y prevenir la violencia" (Garaigordobil, 2010, p.292). En este programa se desarrollan una serie de dinámicas yactividades en las que se debaten temas relacionados con la paz y la resolución de conflictos humanos. El estudio realizado por Garaigordobil (2010) pone de manifiesto los resultados positivos de la implantación de este proyecto. 
Existe en nuestro país la Asociación Española para la Prevención del Acoso Escolar (AEPAE), impulsora y promotora del "Plan Nacional contra el Acoso Escolar", cuyo objetivo es "desarrollar en niños y adolescentes un comportamiento preventivo dirigido a la resolución de conflictos en el entorno escolar" (AEPAE, s. f., párr. 2). Este plan tiene cuatro fases: test TEBAE (Test de evaluación breve del acoso escolar); campaña de sensibilización en aulas y con familias; intervención y seguimiento a través de dinámicas entre iguales con ayuda del profesorado; y protocolo de actuación en colegios (en función de cada comunidad autónoma).

En esta misma línea, se encuentra la "Asociación Postbullying", la cual actúa y ofrece ayuda ante casos de bullying, y asesora y orienta a aquellas familias víctimas de acoso escolar a través de ejercicios virtuales y recursos digitales. Además, se encuentran en su página web proyectos con los que trabajar la educación para la paz en las aulas.

Otro de los programas centrados en la mejora de la integración escolar y el trabajo de la escuela inclusiva y no violenta en nuestro país es el "TEl" (Tutoría entre iguales). Iniciado en 2002, "se basa en la tutorización emocional entre iguales donde el respeto, la empatía y el compromiso son los pilares básicos de su desarrollo en los centros educativos" (González, 2016, párr. 2); algo que, sin duda, es necesario trabajar para la prevención de bullying.

Podemos asegurar que son muchos los programas que existen y están a nuestro alcance con el objetivo de prevenir casos de violencia escolar. Muchos estudios han concluido que estos proyectos tienen consecuencias positivas en su implantación. Estos planes se fundamentan en la prevención del acoso escolar, en su seguimiento e intervención para lograr, a través de un profundo trabajo de sensibilización, acabar con este fenómeno.

La bibliografía especializada muestra cómo una de las formas más potentes de trabajar esta sensibilización es mediante el "método socioafectivo".

Los países nórdicos fueron pioneros en ejecutar proyectos de educación para la paz; pero tras algunos años de puesta en marcha, se evaluó su impacto y se observó que cuando se preguntaba al alumnado sobre cifras, informes, tratados, etc. relacionados con la paz, las respuestas eran positivas. Por el contrario, cuando se les preguntaba su opinión acerca de situaciones de vulnerabilidad en determinados países del mundo, sus respuestas eran del tipo: "que suerte que no vivo ahí", "menos mal que yo tengo de todo", etc. Por lo tanto, se observó que "Ios niños llegaron a conocer con lujo de detalles las razones del desequilibrio Norte-Sur, pero esto no cambió su actitud vital" (Aguilera, Gómez, Morollón y de Vicente, 2009, p. 42). Es decir, no había habido un cambio de actitud en la niñez con la que se había trabajado la idea de paz. “De esta experiencia se desprendió la importancia de no sólo introducir contenidos, sino de poner un énfasis especial en otros apartados de la educación: actitud del profesorado, organización escolar y la metodología con la que se impartían esos [contenidos]" (Cascón, 2008, p. 230). 
doi: http://dx.doi.org/10.15359/ree.23-3.3

URL: http://www.una.ac.cr/educare

CORREO: educare@una.cr

Es en este punto se hace necesario acercar al estudiantado "a la realidad ... donde se dan las desigualdades ... con la finalidad de que tengan una visión crítica" (Hamodi y Contreras, 2018, p. 188). Para ello, se debe "provocar un distanciamiento de sí mismos para verse desde fuera" (Ruiz de Lobera, 2004, p. 71) y "resocializar esquemas mentales, trabajando la vertiente emocional-actitudinal-moral" (Jordán Sierra et al., 2004, p. 19).

Diferentes trabajos (Aguilera, et al., 2009; Cascón, 2008; Hamodi y Contreras, 2018; Jares 1995, 2004; UNESCO, 1983; Vidal y Caireta, s. f.) abogan por la utilización de este método que busca potenciar lo afectivo y lo vivencial a la hora de abordar temas o problemas sociales. Este enfoque no desprecia lo cognitivo, sino que lo complementa con lo emocional y empático, al permitir que, en el proceso educativo, la persona tome parte en su totalidad, con el objetivo de pasar por estas fases: sentir, pensar, actuar.

\section{Metodología}

La metodología utilizada en el presente trabajo es mixta (cualitativa y cuantitativa), a pesar de que se plantea la sociometría (test sociométrico) como un área cuantitativa de la sociología, fundamental para la detección de posibles casos de bullying al permitir conocer las redes de relaciones interpersonales en un grupo. No obstante, se han incluido técnicas de investigación cualitativa porque, como sugiere Gómez (2013, p. 864), "sería recomendable incorporar trabajos cualitativos, o que combinen ambas perspectivas, con la finalidad de contar con diferentes aproximaciones y tener mayor información acerca del fenómeno".

La experiencia se llevó a cabo en un colegio público localizado en una zona de amplia expansión demográfica, en la que residen personas de todo tipo de edades, con predominio de población adulta (joven) con hijos e hijas en edad escolar. Las características económicas y socioculturales de la zona son muy variadas. No existe un claro predominio de ninguna clase social, de hecho, se pueden observar distintos tipos de niveles económicos y socioculturales: alto, medio y bajo. Cabe destacar el aumento de estudiantes inmigrantes que ha experimentado la escuela en estos últimos años, alguna cantidad con importantes carencias en lenguaje. El nombre y la localidad del centro no se hacen públicos para garantizar el absoluto anonimato, siguiendo el código ético de la investigación.

La muestra de participantes engloba a una clase de 19 estudiantes de $4^{\circ}$ de primaria. Se decidió hacerlo en esta aula, en primer lugar, debido al desarrollo de la práctica docente en esta misma, y en segundo lugar porque "es una etapa de la educación obligatoria con muchísima relevancia, caracterizada por la creación de identidades sociales y personales ... Se produce un fuerte proceso en ... la socialización del alumnado, a través de la incorporación de normas, valores y creencias" (Hamodi, 2014, p. 37); y además, porque se trata de un curso crucial en el que, por lo general, se ha percibido cierto aumento de casos de bullying (Eslea y Rees, 2001). 
La investigación se ha desarrollado en tres fases, cada una de ellas con unos objetivos específicos. A continuación, se enumeran las fases y se detalla la metodología utilizada en cada una de las fases (las técnicas de investigación, herramientas de análisis, etc.).

\section{Fase: Exploración del contexto}

Los instrumentos utilizados en esta fase fueron:

- Guía de observación. Es un instrumento que sirvió para sintetizar y sistematizar el proceso, la duración y frecuencia de los registros. Resultó necesario apoyarnos en esta guía para registrar de manera uniforme y metódica la observación sistemática en diferentes momentos: recreos, actividades grupales dentro y fuera del aula y pasillos del colegio. Esta guía estuvo compuesta por:

- Un primer cuadro donde se recogieron los elementos identificativos (nombre del observador, fecha, horario, lugar y tipología de la actividad que se está observando).

- Una lista con diferentes categorías de análisis: 1. No quieren participar. 2. Niños aislados o niñas aisladas. No les dejan participar otros compañeros o compañeras. 3. Conducta rebelde, rol autoritario. 4. Conducta pasiva o inhibida. 5. Toma parte activa en los juegos con sus pares. 6. Capacidad para ponerse en el lugar del otro ser, empatía y asertividad. 7. Incapacidad empática y asertiva. 8. Aprende de los demás, acepta sus comentarios, y participa en el grupo. 9. No aprende de las demás personas, no acepta sus comentarios, y no participa en el grupo. 10. Capacidad para expresar sus propios sentimientos y adaptarlos a diversas situaciones (inteligencia intrapersonal) 11. Incapacidad para expresar sus propios sentimientos y adaptarlos a diversas situaciones (inteligencia intrapersonal) 12. Se esconde de las demás personas. 13. Deambula de un grupo al otro. 14. Capacidad para resolver conflictos de forma pacífica y cooperativa. 15. Incapacidad para resolver conflictos de forma pacífica y cooperativa. Estas categorías fueron seleccionadas con el fin de dar alcance al cumplimiento de los objetivos planteados en esta investigación.

- Una tabla para rellenar con el nombre de cada estudiante y la respectiva categoría de evaluación.

- Diario de campo. "Los diarios de campo son textos escritos en los que el científico/a y/o profesional, ... registra aquellos acontecimientos que transcurren en el día a día de su experiencia profesional o académica y que le resultan especialmente significativos" (Albertín, 2007, p. 14). Siguiendo esta definición, nuestro diario fue utilizado como un instrumento de investigación subjetivo, con el objetivo de recopilar todos aquellos comportamientos, emociones, impresiones, actitudes, reacciones, etc. del grupo. El diario también recoge aspectos informativos en función de los focos de interés de 
doi: http://dx.doi.org/10.15359/ree.23-3.3

URL: http://www.una.ac.cr/educare

CORREO: educare@una.cr

nuestra investigación; y hechos reflexivos subordinados a la propia experiencia de quien investiga. Este instrumento fue utilizado desde el inicio de la investigación hasta su fin, siempre que las investigadoras tenían acceso a las clases del grupo. En definitiva, "el fundamento del diario de campo está ... ahí: probablemente es el instrumento de investigación óptimo para recoger al propio investigador, por un lado, y, por otro, para captar la investigación como situación" (Velasco y Díaz, 1997, p. 51).

- Test sociométrico. Permite obtener información relevante y adicional a la observación, para examinar la estructura socioafectiva del aula, ya que para prevenir el bullying (fenómeno grupal) se hace imprescindible el análisis de las relaciones sociales entre escolares (Cerezo, 2006). Por ello, se consideró esencial llevar a cabo un test sociométrico al principio de la investigación, ya que este trata de medir aspectos concretos de la estructura grupal mediante el análisis del tipo de elecciones de personas que se realizan en un grupo determinado, según las condiciones que se plantean a la totalidad de miembros (Pineda et al., 2009) y es que "desde muy pequeños, los individuos son capaces de seleccionar conscientemente a las personas" de las que quieren rodearse (Rodríguez, 2001, p. 44). Fue aplicado al inicio de la investigación con el fin de obtener información relevante y adicional a la observación, para examinar la estructura socioafectiva del aula, ya que:

Esta herramienta será útil para el profesor dado que a través de la misma, se puede generar un clima idóneo para el aprendizaje y bienestar general en el aula; puntualizar los individuos que necesitan ayuda y/o contención por la falta de integración a algún grupo o la apatía generada en diversos problemas personales. (Kuz y Falco, 2013, p. 2)

Este método de evaluación se inserta dentro del esquema propuesto por Monjas (2000) como un instrumento de análisis que depende de la fuente de información conocida como "heteroinformación", es decir, aquella obtenida de iguales. Dicha información, en el caso del bullying se orienta a lo siguiente:

Persigue dos objetivos decisivos en la dinámica de la intimidación: por una parte, saber de los sujetos y la asignación de roles dentro del grupo como agresores/as, víctimas testigos, ayudantes del agresor, defensores de la víctima, etc.; y por otra, saber de la posición de aceptación y rechazo de los participantes en las situaciones de intimidación dentro del grupo. (Avilés, 2006, p. 204)

Teniendo en cuenta las características de este método, se realizó una batería de preguntas de dominio académico y social tanto positivo como negativo, con las que además de obtener referencias cuantitativas (elección y rechazo), también alcanzamos datos cualitativos (el porqué de la elección o rechazo) (ver Apéndice A).

8

Carolina Hamodi-Galán y Yara de Benito-Brunet

Los artículos de la Revista Electrónica Educare del Centro de Investigación y Docencia en Educación de la Universidad Nacional, Costa Rica, se comparten bajo términos de la Licencia Creative Commons: Reconocimiento, No Comercial, Sin Obra Derivada 3.0 Costa Rica. Las autorizaciones adicionales a las aquí delimitadas se pueden obtener en el correo: educare@una.cr 
En cada una de las cuestiones, el alumnado tenía que responder escribiendo tres nombres de compañeros o compañeras que atendieran a lo que se requería en los enunciados de las preguntas (se estableció un número fijo de preferencias debido a los objetivos del análisis). Además, debían justificar con argumentos sólidos (teniendo en cuenta que son estudiantes de $4^{\circ}$ de primaria) el porqué de sus elecciones.

Siguiendo esta línea, se pretendió conocer: a) cohesión y cooperación en el grupo; b) relaciones interpersonales; c) posición de cada miembro con respecto al resto; d) nivel de aislamiento o rechazo; e) grado de sociabilidad.

Los datos del test sociométrico fueron trasladados, a través de la herramienta online "Sometics", a una matriz sociométrica (tabla de doble entrada) que permite cuantificar los resultados referentes a las relaciones interpersonales del grupo. De esta forma, fue posible visualizar las conexiones y relaciones sociales del grupo-clase en dos dominios: académico y social. Las respuestas recogidas fueron trasladadas a la aplicación digital que las vuelca de manera automática en una matriz sociométrica. Se realizó de forma online, ya que se trata de una herramienta digital que permite agilizar el proceso y facilitar la fase de análisis de resultados (Van Den Berg y Gommans, 2017).

Cabe señalar que la fiabilidad de este instrumento fue contrastada con los datos resultantes de la observación durante nuestra investigación. Gimeno (1976, p. 106) declara que "muchos investigadores han constatado una fuerte correspondencia entre los datos del test sociométrico y la observación directa y sistemática de los grupos".

\section{Fase: Diseño y desarrollo de una propuesta de intervención para la prevención del bullying}

La propuesta de intervención tiene el objetivo principal de prevenir posibles casos de bullying. En ella, se integran varias dinámicas con las que se trabajan distintas habilidades sociales, empatía, conocimiento dela propia persona, relaciones interpersonales, tomando como marco de referencia el enfoque socioafectivo. Se trata de un método que "parte del trabajo de la empatía, el sentimiento de concordancia y correspondencia con el otro, que permite desarrollar seguridad y confianza en uno mismo, así como habilidad comunicativa verbal y no verbal" (Vidal y Caireta, s. f., p. 6).

Dicha propuesta contiene una serie de dinámicas y actividades grupales cuyo objetivo, en primer lugar, es la prevención del acoso escolar. No obstante, lleva implícito el desarrollo de habilidades sociales. Estas dinámicas fueron establecidas en función de los resultados obtenidos tras el test sociométrico y cuestionarios, además de la observación continua del grupo. 
doi: http://dx.doi.org/10.15359/ree.23-3.3

URL: http://www.una.ac.cr/educare

CORREO: educare@una.cr

La falta de empatía provocada por la dificultad para reconocer los sentimientos y emociones propias; la desensibilización; la carencia de pautas educativas y de responsabilidad para resolver conflictos; el déficit de comunicación como base para la confianza y la autonomía de acción; así como la incapacidad para el trabajo cooperativo son causas principales de bullying (Avilés, 2006). Por todo ello, y teniendo en cuenta los rasgos y características principales de nuestro grupo participante, elaboramos una propuesta para la prevención del acoso escolar apoyada sobre el método socioafectivo. Este, según la UNESCO (1983, p. 106):

Se basará esencialmente en una experiencia -o situación empírica- que el individuo comparte en tanto que miembro de un grupo y que analizará y describirá seguidamente hasta llegar a formular su vivencia, sus actitudes, sus reacciones y hasta tener conciencia de sí mismo, para pasar luego de la descripción al análisis hasta llegar por último a generalizar y extender la experiencia vivida a situaciones exteriores, es decir a situaciones de la vida real.

De esta forma, las dinámicas que se plantearon a través de este método llevaban consigo la búsqueda del aumento de las relaciones interpersonales, así como el autoconocimiento y el desarrollo de la empatía y la implicación del alumnado en la resolución de conflictos. Por ello, se llevaron a cabo siguiendo una serie de pasos propios de este enfoque (Jares, 1995):

1. Vivenciación de una experiencia (real o simulada).

2. Descripción y análisis de esta.

3. Contraste y generalización de la experiencia vivida a situaciones de la vida real.

Como se ha explicado en la fundamentación teórica, el método socioafectivo utilizado en esta investigación busca desarrollar un cambio de actitudes y valores, y por ello se utiliza para la sensibilización sobre problemáticas sociales. En el caso del bullying, no serviría de nada que el estudiantado conociese muy bien en qué consiste el fenómeno, si no se sintiese identificado con el mismo, y no fuese capaz de desarrollar empatía con respecto a las personas que lo sufren. Por ello, el primer paso del método consiste en realizar una dinámica en la que puedan ponerse realmente en la situación de esas personas en situación de vulnerabilidad. Ha de buscarse el alcance de la empatía. Es muy importante diseñar bien las dinámicas y asegurarse de que estas van a producir un cierto cambio al provocar sensaciones y sentimientos, pues es así como podemos empezar a trabajar sobre el desarrollo de la empatía. En ocasiones se requiere de la realización de varias dinámicas para ello. Una vez que se ha desarrollado la dinámica y ha habido "emociones", Ilega la segunda fase, que consiste en describir lo que ha sucedido y exponer los sentimientos. Es clave preguntar al estudiantado cómo se han sentido durante el desarrollo de la dinámica. Finalmente, 
en la última fase, se busca el alcance de conclusiones, contrastando y generalizando la experiencia o extrapolándola a otras situaciones de la vida. Es en este punto donde debemos hacer énfasis sobre las situaciones de vulnerabilidad sobre las que queremos trabajar.

En las dinámicas desarrolladas a través del método socioafectivo, los 'bloques de contenido' sobre los que trabajamos fueron los 5 siguientes:
a) Distorsión de la información
b) Debate - aceptación de opiniones - respeto
c) Confianza - pensamiento subjetivo
d) Trabajo de emociones
e) Resolución de conflictos

\section{3a Fase: Evaluación del impacto de la propuesta}

El análisis del efecto de la propuesta se llevó a cabo durante la última semana de la experiencia, a través de la observación y anotación de reacciones en el diario de campo; además, el alumnado rellenó un informe de evaluación de la propuesta (ver Apéndice B) que contiene una serie de ítems que evaluar: del 1 (nada) al 4 (totalmente). Este sirvió, por un lado, para obtener información directamente de participantes $\mathrm{y}$, por otro, para comprobar aquellos aspectos que podían ser mejorados.

A través de esta 'autoevaluación', según Aranda (2010, p. 7) "evaluación que una persona realiza sobre sí misma o sobre un proceso y/o resultado personal", pretendimos promover entre el alumnado el pensamiento crítico y constructivo y el desarrollo de su autonomía y responsabilidad.

\section{Resultados}

El test sociométrico arroja una matriz sociométrica (Tabla 1). Por un lado, nos centraremos en los datos relativos al dominio académico, es decir, la cantidad de elecciones y rechazos que han sido obtenidos en cuanto a cohesión y cooperación del grupo-clase. Las preguntas que tuvieron que contestar para ello, están relacionadas con el trabajo desempeñado en el aula:

1. ¿A qué compañeros o compañeras elegirías para trabajar en clase?

2. ¿A qué compañeros o compañeras NO elegirías para trabajar en clase? 
doi: http://dx.doi.org/10.15359/ree.23-3.3

URL: http://www.una.ac.cr/educare

CORREO: educare@una.cr

Tabla 1: Matriz sociométrica "Dominio académico"

\begin{tabular}{|c|c|c|c|c|c|c|c|c|c|c|c|c|c|c|c|c|c|c|c|c|c|}
\hline 1 & 飞 & $\sum_{<}$ & ऐัे & 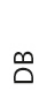 & 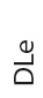 & $\begin{array}{l}\bar{\nu} \\
\text { ¿े }\end{array}$ & 樆 & $\sum_{L}^{\xi}$ & 吕 & 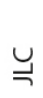 & 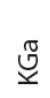 & $\sum$ & $\stackrel{̊}{9}$ & $\begin{array}{l}\frac{\overline{\bar{v}}}{\frac{\mathrm{M}}{\Sigma}} \\
\end{array}$ & $\sum_{n}^{0}$ & $\begin{array}{l}\text { స్ } \\
\text { }\end{array}$ & $\mathscr{F}$ & $\sum_{5}^{\pi}$ & $\stackrel{\Xi}{\rightleftharpoons}$ & + & I \\
\hline AJ & $\mathrm{x}$ & V & V & & & & & & & $x$ & & V & & & & $\mathrm{x}$ & & $x$ & & 3 & 3 \\
\hline $\mathrm{AM}$ & & $\mathrm{x}$ & & & & v & $x$ & & & & & & & v & $x$ & & & $x$ & v & 3 & 3 \\
\hline ASor & & $\mathrm{x}$ & $x$ & & & & & & & $x$ & & & & v & v & & & $x$ & V & 3 & 3 \\
\hline DB & $\mathrm{x}$ & V & & $x$ & v & & & & & $x$ & & V & & & $x$ & & & & & 3 & 3 \\
\hline DLe & $\mathrm{x}$ & V & & v & $x$ & & $\mathrm{x}$ & & & & & v & & & & & & $x$ & & 3 & 3 \\
\hline DPel & & V & & & & $x$ & & & V & $x$ & & $\mathrm{~V}$ & & & $x$ & & & $x$ & & 3 & 3 \\
\hline ESar & & $\mathrm{x}$ & & & & & $\mathrm{x}$ & v & & $\mathrm{x}$ & & $\mathrm{x}$ & & & & V & & & V & 3 & 3 \\
\hline FMJim & & $x$ & & & $x$ & v & & $x$ & & v & & & & & & & v & $x$ & & 3 & 3 \\
\hline $\mathrm{HBa}$ & $\mathrm{x}$ & V & & v & & v & & & $\mathrm{x}$ & & & & $x$ & & $x$ & & & & & 3 & 3 \\
\hline $\mathrm{JLC}$ & & & & & $x$ & $x$ & $\mathrm{x}$ & V & & $x$ & & & & & & & V & V & & 3 & 3 \\
\hline KGa & $x$ & V & & & & & & & & & $x$ & $\mathrm{~V}$ & v & & $x$ & & & $x$ & & 3 & 3 \\
\hline LM & $\mathrm{x}$ & & V & & & & $\mathrm{x}$ & & & & & $x$ & v & & & & & $x$ & v & 3 & 3 \\
\hline LRo & & & V & & $x$ & & & $x$ & & & & & $x$ & v & & & $x$ & & V & 3 & 3 \\
\hline MEHer & & & & & $x$ & & & $x$ & & & & $\mathrm{~V}$ & & $x$ & V & & & $\mathrm{x}$ & V & 3 & 3 \\
\hline SMol & & & V & & & & & $x$ & $\mathrm{x}$ & & & v & & & $x$ & v & & $x$ & & 3 & 3 \\
\hline SSan & & & & & & & V & V & & & & & $x$ & & V & $x$ & $\mathrm{x}$ & $x$ & & 3 & 3 \\
\hline TTs & $\mathrm{x}$ & & $x$ & & V & & & v & & V & & & & & $\mathrm{x}$ & & $\mathrm{x}$ & & & 3 & 3 \\
\hline UMa & $x$ & & & & & & & $x$ & & & & $\mathrm{~V}$ & $\mathrm{~V}$ & & $x$ & & & $x$ & V & 3 & 3 \\
\hline YLu & & & & & & v & & & & $x$ & & $\mathrm{~V}$ & $\mathrm{~V}$ & & $x$ & & & $x$ & $x$ & 3 & 3 \\
\hline Total positiva & 0 & 6 & 4 & 2 & 2 & 4 & 1 & 4 & 1 & 2 & 0 & 9 & 4 & 3 & 3 & 2 & 2 & 1 & 7 & 57 & \\
\hline Total negativa & 7 & 3 & 1 & 0 & 4 & 1 & 4 & 4 & 1 & 6 & 0 & 1 & 2 & 0 & 8 & 1 & 2 & 12 & 0 & & 57 \\
\hline
\end{tabular}

Nota: Elaboración propia a partir de la herramienta online Sometics.

A partir de los umbrales que se establecen en estas puntuaciones y de la comparación de diferentes índices, se puede clasificar al alumnado de un aula en diversos tipos o estatus; siguiendo a Fernández (2013) en: popular, rechazado, ignorado, promedio y controvertido. 
En la Tabla 1, podemos observar casos bastante llamativos. En cuanto a las selecciones positivas, destacan dos alumnas con 9 y 7 puntos respectivamente, de las cuales solo una ha recibido una nominación negativa. En este caso, podríamos clasificar a estas estudiantes como "populares", ya que gustan a la mayoría del grupo.

En contraposición, destaca un miembro del grupo por encima de los demás con 12 selecciones negativas y ninguna positiva; además de otro con 7 negativas y ninguna positiva. Siguiendo la clasificación citada, situaríamos a estos estudiantes dentro del perfil de "rechazado", correspondiéndose con estudiantes que no resultan agradables para la mayoría de sus iguales.

Observamos también en los resultados de la aplicación del test sociométrico un caso que no ha obtenido ni rechazos ni elecciones. Según la clasificación anteriormente señalada se incluiría dentro de la categoría "ignorado". Se trata de quienes:

resultan indiferentes para su grupo de iguales, reciben poca atención de éstos y son muy poco conocidos. ... [Suelen ser] pacíficos, tímidos y reservados, pero no tienen por qué estar aislados socialmente como algunos de los adolescentes rechazados. Aunque demuestran menos sociabilidad que los iguales promedio, respetan las reglas y están comprometidos en actividades socialmente aceptadas, aunque en grado menor que los niños más aceptados y de forma más aislada. (Fernández, 2013, p. 139)

En cuanto al resto del grupo, vemos que los resultados son por lo general, bastante equitativos, es decir, existe una correlación entre quienes se eligen de forma positiva entre sí, $y$ quienes lo hacen negativamente. El alumno " $x$ " ha elegido de forma positiva a " $y$ " e " $y$ " ha elegido a " $\mathrm{x}$ " positivamente también; lo mismo ocurre con las puntuaciones negativas.

Por lo que corresponde a los 'negativo-positivo' (el alumno " $x$ " ha elegido positivamente a "y", pero " $y$ " le ha rechazado) encontramos tres casos.

Señalamos un 'caso conflictivo' (denominado así por el propio programa informático), ya que ha recibido el mismo número de nominaciones positivas como negativas.

Hay que mencionar, además, que en ambas preguntas se pidió una respuesta más 'cualitativa', el alumnado tuvo que argumentar el porqué de sus preguntas. Este hecho, nos dio muchas pistas relacionadas con la observación simultánea que se estaba llevando a cabo. Las respuestas gran cantidad coincidían no solo en las selecciones de forma negativa, sino también en las respuestas positivas:

- "Porque están siempre molestando y gastando el tiempo"

- "Porque no trabajan y no dejan concentrarse"

+ "Porque hacen siempre los deberes y ayudan a los demás"

+ "Porque son muy trabajadores y me caen bien" 
doi: http://dx.doi.org/10.15359/ree.23-3.3

URL: http://www.una.ac.cr/educare

CORREO: educare@una.cr

Por otro lado, obtuvimos datos relacionados con el dominio social (ver Tabla 2):

+ ¿A cuáles de tus compañeros o compañeras invitarías a tu cumpleaños?

- ¿A cuáles de tus compañeros o compañeras NO invitarías a tu cumpleaños?

Tabla 2: Matriz sociométrica "Dominio social"

\begin{tabular}{|c|c|c|c|c|c|c|c|c|c|c|c|c|c|c|c|c|c|c|c|c|c|}
\hline 1 & 飞 & $\sum_{<}$ & के & คี & $\stackrel{\oplus}{\Delta}$ & $\begin{array}{l}\bar{v} \\
\overline{0}\end{array}$ & 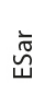 & $\sum_{\longleftarrow}^{\xi}$ & 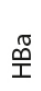 & 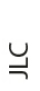 & $\begin{array}{l}\mathbb{N} \\
\stackrel{0}{ }\end{array}$ & $\sum$ & $\stackrel{0}{\simeq}$ & $\begin{array}{l}\frac{\bar{d}}{T} \\
\stackrel{\Psi}{\Sigma}\end{array}$ & $\sum_{n}^{\overline{0}}$ & $\underset{\sim}{\tilde{N}}$ & $\stackrel{\curvearrowleft}{E}$ & $\sum_{J}^{\pi}$ & $\stackrel{\Xi}{\check{\nu}}$ & + & 1 \\
\hline AJ & $x$ & & & & & & & V & & & V & & $x$ & & $x$ & & V & $x$ & & 3 & 3 \\
\hline$A M$ & & $x$ & & & & V & $x$ & & V & & V & & & & $x$ & & & $x$ & & 3 & 3 \\
\hline ASor & & & $x$ & & & V & & $x$ & $x$ & & & $x$ & V & & & & & & V & 3 & 3 \\
\hline DB & & & & $x$ & & & $x$ & & V & & V & & V & & & $x$ & & $x$ & & 3 & 3 \\
\hline DLe & & $x$ & & V & $x$ & & & V & $x$ & & $x$ & & & & & & V & & & 3 & 3 \\
\hline DPel & & & V & & & $x$ & & V & & $x$ & & & & & $x$ & & & $x$ & V & 3 & 3 \\
\hline ESar & $x$ & & & V & & & $x$ & & & & $x$ & & & & & V & & $x$ & V & 3 & 3 \\
\hline FMJim & V & & & & & V & & $x$ & & & & $x$ & $x$ & & $x$ & & V & & & 3 & 3 \\
\hline $\mathrm{HBa}$ & $x$ & V & $x$ & V & & & & & $x$ & & V & & & & $x$ & & & & & 3 & 3 \\
\hline JLC & $x$ & & & & & & $x$ & & & $x$ & & & V & V & & & V & $x$ & & 3 & 3 \\
\hline KGa & & V & & & & & $x$ & & & $x$ & $x$ & V & V & & & & & $x$ & & 3 & 3 \\
\hline LM & & & V & $x$ & $x$ & & & & & & & $x$ & V & & & & & $x$ & V & 3 & 3 \\
\hline LRo & $x$ & & V & & & V & $x$ & & & & & & $x$ & V & & & $x$ & & & 3 & 3 \\
\hline MEHer & & & & & $x$ & & V & $x$ & & & & V & V & $x$ & & & & $x$ & & 3 & 3 \\
\hline SMol & & & V & & & & & $x$ & $x$ & & & V & & & $x$ & V & & $x$ & & 3 & 3 \\
\hline SSan & $x$ & $x$ & & V & & $x$ & & & & & & V & & & & $x$ & & & V & 3 & 3 \\
\hline TTs & & & $x$ & & V & & & & $x$ & V & & V & $x$ & & & & $x$ & & & 3 & 3 \\
\hline UMa & $x$ & & $x$ & & & & & & & V & & & V & & $x$ & & & $x$ & V & 3 & 3 \\
\hline YLu & & & & $x$ & $x$ & V & & & & & & V & V & & $x$ & & & & $x$ & 3 & 3 \\
\hline Total positiva & 1 & 2 & 4 & 4 & 1 & 5 & 1 & 3 & 2 & 2 & 4 & 6 & 8 & 2 & 0 & 2 & 4 & 0 & 6 & 57 & \\
\hline Total negativa & 6 & 2 & 3 & 2 & 3 & 1 & 5 & 3 & 4 & 2 & 2 & 2 & 3 & 0 & 7 & 1 & 1 & 10 & 0 & & 57 \\
\hline
\end{tabular}

Nota: Elaboración propia a partir de la herramienta online Sometics. 
Como podemos observar, en la matriz sociométrica de dominio social destaca una alumna con 6 elecciones positivas que no ha sido seleccionada negativamente; esta persona es la misma a la que nadie ha rechazado en las preguntas de dominio académico, y coincide en ambos dominios con su categorización de "popular". Destacamos también que los focos de selección negativa son claramente visibles: hay dos alumnos que no han sido seleccionados de forma positiva ninguna vez. Por lo general, quienes han recibido elecciones negativas, en su totalidad, tienen una puntuación extremadamente baja en positivas y viceversa coincidiendo en ambos dominios con su categorización de "rechazado".

Al resto de estudiantes podríamos situarlo en la categorización de "promedio". Cabe señalar que no se encuentra en el grupo de manera muy marcada ningún estudiante "controvertido", entendiéndolo como aquel que tiene "elevados índices de aceptación y de rechazo" (Fernández, 2013, p. 140).

En este caso, hay más estudiantes que se han elegido mutuamente de forma positiva, en comparación con quienes lo hicieron negativamente. Se dan cuatro situaciones 'negativopositivo" en las que el alumno " $x$ " ha seleccionado al " $y$ "; pero " $y$ " ha rechazado a " $x$ ". No se ha detectado ningún 'caso conflictivo'.

En cuanto al porqué de las respuestas positivas, encontramos más variedad en sus contestaciones:

+ "Son muy majos y se portan bien conmigo"

+ "Ellos me han invitado a su cumpleaños"

+ "Me ayudan en lo que necesito"

+ "Son muy simpáticos"

+ "Nunca me han dado la espalda"

+ "Tengo más confianza con ellos"

Mientras que en las negativas, las respuestas son muy similares a las del dominio académico; están interrelacionadas:

- "Porque siempre molestan y no hacen nada"

- Porque no me tratan bien y algunos son muy pesados"

- "Porque no los conozco bien"

La última pregunta de este test está relacionada con los 'roles'y las percepciones que cada estudiante tiene sobre sus pares (ver Tabla 3). 
doi: http://dx.doi.org/10.15359/ree.23-3.3

URL: http://www.una.ac.cr/educare

CORREO: educare@una.cr

Tabla 3: Selección de'roles' en el aula

\begin{tabular}{|c|c|c|c|c|c|c|c|c|c|c|c|c|c|c|c|c|c|c|c|}
\hline \begin{tabular}{|c|} 
Estudiante \\
IItem
\end{tabular} & 1 & 2 & 3 & 4 & 5 & 6 & 7 & 8 & 9 & 10 & 11 & 12 & 13 & 14 & 15 & 16 & 17 & 18 & 19 \\
\hline $\mathrm{a}$ & & 4 & 1 & & 2 & 2 & & 1 & & 1 & & 5 & 2 & & & & & & 1 \\
\hline b & & & & & & & 1 & & & & & & & & 10 & 3 & 2 & 3 & \\
\hline c & & 10 & & & & & & & & & & 1 & & & & & & & 8 \\
\hline d & 1 & & 2 & & & & & & 2 & & & & & 9 & & & 4 & \\
\hline e & & 1 & & 3 & & 1 & & 2 & & 1 & 1 & 2 & 1 & & & 1 & & 1 & 3 \\
\hline f & 4 & 1 & 1 & & 2 & 2 & 1 & 1 & & 1 & 1 & & & & & 1 & 1 & 3 & \\
\hline g & & 4 & 3 & & & 2 & & & 1 & 3 & 1 & & & & & 2 & 1 & & 2 \\
\hline h & 1 & & & 1 & 1 & & 2 & & & & & & & & 2 & 2 & 2 & 8 & \\
\hline i & & 2 & 3 & & & & & & 3 & & & & 6 & & 2 & & & 2 \\
\hline j & 1 & 1 & & & & & & 1 & & 2 & 1 & & & 7 & 1 & & 5 & \\
\hline
\end{tabular}

Nota: Elaboración propia.

Los datos obtenidos permitieron observar cómo las respuestas de las matrices sociométricas corresponden con las elecciones del alumnado en muchas de las opciones. Por ejemplo, es destacable que la alumna 19 no ha sido nuevamente elegida de forma negativa por nadie, es más, siempre lo ha sido de modo positivo, tal y como hemos podido apreciar anteriormente. Según la bibliografía especializada (Fernández, 2013, p. 139), "la popularidad puede atribuirse a distintos criterios, como el prestigio (por razones académicos, destreza en los deportes, etc.), aspectos emocionales y relativos a su relación con el grupo". Se trata de estudiantes que "muestran una mayor competencia social y habilidades cognitivas, una menor conducta agresiva y disruptiva, así como menos sentimientos de soledad, en comparación con los otros tipos sociométricos".

Por otro lado, se repite el esquema previo, es decir, los focos de atención en los ítems negativos vuelven a coincidir. La bibliografía especializada indica que suele tratarse de estudiantes con las siguientes condiciones:

No resultan agradables para la mayoría de sus iguales. Estos adolescentes se implican con mayor frecuencia en comportamientos violentos, disruptivos o que conlleven una violación de las reglas institucionales, informan de relaciones más conflictivas con otros compañeros y profesores y presentan una baja competencia social y académica. Diferentes 
estudios muestran, sin embargo, que no todos los adolescentes rechazados participan en actos violentos y que, por tanto, la violencia no se asocia necesariamente al rechazo. Existe una proporción de alumnos rechazados que muestran un excesivo retraimiento social, depresión y ansiedad, hecho que también puede contribuir a que el adolescente sea rechazado, y a que permanezca en este tipo sociométrico. En definitiva, los adolescentes rechazados parecen responder a diferentes perfiles, que reflejan la heterogeneidad de este tipo sociométrico. (Fernández, 2013, p. 139)

Los roles popularidad, eficacia en el trabajo, simpático, etc. son incluso más visibles en esta tabla. Sobresale una alumna que, a pesar de no haber sido nominada por nadie en la mayoría de ítems, sí lo ha sido de forma excepcional en la letra i) su capacidad para resolver conflictos.

Con respecto a estos resultados y nuestra observación, se tuvieron en cuenta todos los roles señalados anteriormente. Es decir, no solo se consideró para la creación de la propuesta el alumnado elegido de forma negativa, sino tanto el que lo había sido de forma positiva (con el fin de crear grupos heterogéneos) y quienes habían pasado sin advertirse tanto de manera positiva como negativa.

Cabe señalar, además, que resultó imprescindible trabajar la resolución de problemas y el tratamiento de la información (rumores). En cuanto a las relaciones interpersonales, destaca que el problema principal radica en que es un grupo recientemente formado, se conocieron al inicio del presente curso escolar; hecho que hace a la mayoría tener una postura reticente a la hora de conocer más a sus pares. Se refugian en amistades con las que ya habían compartido vivencias y aula. Esta circunstancia nos exigió un trabajo de conocimiento entre iguales, intercambio de experiencias, cualidades, y trabajo de resiliencia; esto último es un aspecto importante, ya que el estudio realizado por Donnon (2010) concluye que estudiantes con un perfil sólido de resiliencia tienen menos comportamientos agresivos y participan menos en situaciones de acoso escolar; sin embargo, quienes presentan habilidades sociales poco desarrolladas poseen más propensión a participar en el bullying.

Por otro lado, se observó el incremento de quejas hacia dos alumnos durante el desarrollo de actividades cooperativas y en una de las tareas por grupos, una cantidad alta mostró insatisfacción al saber que les tocaba trabajar con determinados compañeros o compañeras. Hecho que nos remite directamente a los resultados obtenidos del test sociométrico, donde este grupo de estudiantes es el mismo elegido negativamente para el trabajo en equipo. Esto nos indicó, notoriamente, la necesidad de potenciar la cooperación en el aula, pues "son varios los autores que proponen el aprendizaje colaborativo como medida para que los niños aprendan a trabajar juntos, ayudándose entre ellos y dándose cuenta así de que todos pueden aportar cosas positivas y enriquecedoras a la hora de llevar a cabo una determinada tarea" (Hamodi y Jiménez, 2018, p. 34). De hecho, Avilés (2006) afirma que el trabajo cooperativo no solo mejora la convivencia, sino que también constituye una medida de prevención frente al acoso escolar. 
doi: http://dx.doi.org/10.15359/ree.23-3.3

URL: http://www.una.ac.cr/educare

CORREO: educare@una.cr

En rasgos generales, no observamos ninguna situación de aislamiento; no obstante, coincidiendo con los datos del test sociométrico, si faltaba integración de un par de estudiantes en el grupo. Esto fue trabajado tanto en actividades propias de clase, como en las dinámicas de la propuesta. De tal forma que no se dan situaciones de bullying como tal, ya que no existe un continuo abuso de poder sobre una víctima en concreto, ni son situaciones repetidas en el tiempo; tal como indicábamos en los antecedentes teóricos previos.

Por otro lado, el centro dispone de un protocolo de prevención de bullying que se lleva a cabo en las clases de niveles más altos y que contiene aspectos estrechamente relacionados con el anteriormente mencionado "TEI" a nivel nacional.

Tras la ejecución de la propuesta de prevención de bullying basada en el método socioafectivo (que como se ha indicado anteriormente se compone de tres fases: 1) Vivenciación de una experiencia; 2) Descripción y análisis de la experiencia y 3) Contraste y generalización de la experiencia a situaciones de la vida real) se analizó su impacto. Los resultados muestran los siguientes aspectos: el $100 \%$ del alumnado desea seguir trabajando en este tipo de actividades. Además, considera que es necesario comunicarse adecuadamente para que no surjan problemas de entendimiento y no puedan divulgarse falsos rumores. El mismo porcentaje considera haberse implicado y mantenido interés en las tareas grupales.

En cuanto a las relaciones de grupo, un $50 \%$ (10 estudiantes) cree que han servido totalmente para reforzar su afinidad; un $35 \%$ (7 estudiantes) considera que sus relaciones han mejorado considerablemente; y un 15\% ( 2 estudiantes) indica que sus relaciones siguen estables, ya que no han sido capaces de conocer más profundamente a sus pares.

La empatía ha sido uno de los aspectos que el $70 \%$ del aula (14 estudiantes) ha conseguido desarrollar. Mientras que solo un $35 \%$ ha logrado aplicar lo trabajado en clase a su vida cotidiana.

La última cuestión era abierta. Entre sus respuestas encontramos:

- "Hubiera hecho más actividades asi"

- "Me hubiera gustado repetir más veces las actividades"

- "Me han encantado. Quiero seguir haciendo más juegos"

Los resultados positivos tras la aplicación del método socioafectivo para la prevención del bullying, evidenciadas en la experiencia que se aplicó, coinciden con las evidencias presentadas procedentes de otras prácticas sustentadas sobre este mismo método (Hamodi y de Benito, 2018; Hamodi y Jiménez, 2018), el cual ha sido muy certero en el logro de los objetivos de la investigación que sustenta este artículo. 


\section{Conclusiones}

El fenómeno bullying y su prevención dentro del contexto escolar es un área donde la investigación y la práctica deben ir íntegramente unidas. Así, los grupos docentes necesitan desarrollar prácticas de investigación-acción que permitan conocer el contexto en el que trabajan, para posteriormente intervenir y, finalmente, evaluar las acciones llevadas a cabo.

Siguiendo esta premisa, en primer lugar, se considera imprescindible comenzar por el análisis del contexto del aula y el conocimiento de las relaciones interpersonales que en esta se manifiestan. Para ello, la combinación de técnicas de investigación cuantitativas y cualitativas permite una comprensión holística sobre las relaciones interpersonales del grupo-clase; pero la utilización de la sociometría mediante test sociométrico es fundamental para aproximarse al estudio de estas relaciones de manera exitosa, pues permiten la obtención de datos que no se podrían recoger mediante otras técnicas (como la observación del personal docente). Por lo tanto, es fundamental implicar al estudiantado en el proceso de análisis de sus relaciones interpersonales mediante esta forma de detección. Además, es fundamental establecer este diagnóstico que aporte claves desde las que actuar con sentido y que permitan lograr el éxito de la práctica.

En segundo lugar, es primordial diseñar y ejecutar experiencias que permitan prevenir el bullying, sustentadas en el conocimiento del contexto (obtenido en la fase inicial). Esto nos permitirá detectar sujetos proactivos o líderes para apoyar las acciones positivas, pero también detectar quiénes tienen falta de empatía, provocada por la dificultad para reconocer los sentimientos y emociones propias, la desensibilización, la carencia de pautas educativas y de responsabilidad para resolver conflictos, el déficit de comunicación como base para la confianza y la autonomía de acción, así como la incapacidad para el trabajo cooperativo. Detectar esto es de máxima relevancia, debido a que son causas principales que provocan bullying.

Así, para prevenir el bullying es básico potenciar dos aspectos: la empatía y la cohesión grupal. Para desarrollar la empatía, una metodología muy potente es el método socioafectivo, que permite al estudiantado ponerse en lugar de otras personas al desarrollar conciencia y posibles cambios de actitud. Este enfoque otorga una gran oportunidad para experimentar, reflexionar y actuar; lo que tiene resultados notablemente beneficiosos en tres direcciones: estudiante-estudiante; docente- estudiante; estudiante-docente. Por otro lado, para fomentar la cohesión grupal es imprescindible el trabajo en equipo. Esto puede desarrollarse mediante actividades y tareas diarias encaminadas a la consecución del trabajo cooperativo. No obstante, consideramos imprescindible trabajar de manera transversal (en las diferentes áreas, asignaturas o materias) y de manera prolongada en el tiempo (incluso en diferentes cursos y etapas a lo largo de la educación obligatoria). De esta forma se puede obtener una evolución progresiva positiva en relación con aquellas actitudes y reacciones que puedan darse en situaciones adversas, de exclusión o acoso. 
doi: http://dx.doi.org/10.15359/ree.23-3.3

URL: http://www.una.ac.cr/educare

CORREO: educare@una.cr

En tercer lugar, es esencial la fase de la evaluación del impacto de la experiencia, ya que permite cerrar el ciclo de investigación-acción modificando los aspectos pertinentes para desarrollarla nuevamente (en el mismo u otro contexto). En la experiencia presentada, esta fase mostró los beneficios de trabajar con el método socioafectivo, que permitió desarrollar la capacidad empática en la mayoría de los casos (en diferentes niveles) dentro del grupo, así como maximizar la cohesión grupal y la comunicación.

Finalmente, estimamos necesario apelar a la formación de docentes en estas temáticas y cambiar nuestra actitud ante el acoso escolar y comenzar a ser conscientes de la situación que en nuestra sociedad ocupa este fenómeno, poner en marcha todos los mecanismos inter e intrapersonales para situarlo en su lugar exacto desde la perspectiva social y educativa, y trabajar para su prevención y no solo para su solución cuando el problema ya ha emergido.

\section{Referencias}

Albertín, P. (2007). La formación reflexiva como competencia profesional. Condiciones psicosociales para una práctica reflexiva. El diario de campo como herramienta. Revista de Enseñanza Universitaria, 30, 7-18. Recuperado de http://institucional.us.es/revistas/ universitaria/30/Albertin.pdf

Aguilera, B., Gómez, J., Morollón, M. y de Vicente, J. (2009). Educación intercultural. Análisis y resolución de conflictos ( $7^{\circ} \mathrm{ed}$.). Madrid: Catarata.

Aranda, A. F. (2010). La autoevaluación: Una estrategia docente para el cambio de valores educativos en el aula. Ser Corporal, 3, 6-18. Recuperado de https://dialnet.unirioja.es/ servlet/articulo?codigo $=3441758$

Asociación Española para la Prevención del Acoso Escolar (AEPAE). (s. f.). Es la fundadora e impulsora del plan nacional contra el acoso escolar. Recuperado de https://aepae.es/aepae

Avilés, J. M. (2006). Bullying: El maltrato entre iguales. Agresores, víctimas y testigos en la escuela. Salamanca: Amarú.

Cascón, P. (2008). Educar para la paz. En Secretaría General Técnica (Ed.), Por preguntar que no quede. Libro del profesorado (pp. 211-236). Madrid: Ministerio de Educación y Ciencia. Recuperado de http://www.fundacionfide.org/upload/16/76/por preguntar que no quede libro profe.pdf

Cerezo, F. (1997). Conductas agresivas en la edad escolar. Aproximación teórica y metodológica. Propuesta de intervención. Madrid: Pirámide. 
Cerezo, F. (2006).Violenciay victimización entreescolares. El bullying: Estrategias de identificación y elementos para la intervención a través del Test Bull-S. Revista Electrónica de Investigación Psicoeducativa, 4(9), 333-352. Recuperado de http://www.investigacion-psicopedagogica. org/revista/articulos/9/espannol/Art 9 115.pdf

Donnon, T. (2010). Understanding how resiliency development influences adolescent bullying and victimization. Canadian Journal of School Psychology, 25(1), 101 113. doi: https://doi. org/10.1177/0829573509345481

Echeverri, A., Gutiérrez, R. A., Ramírez, C. M. y Morales, S. A. (2014). Hacia una construcción del concepto violencias escolares. Revista Colombiana de Ciencias Sociales, 5(1), 122-138. Recuperado de http://www.funlam.edu.co/revistas/index.php/RCCS/article/view/971

Eslea, M. y Rees, J. (2001). At what age are children most likely to be bullied at school? Aggressive Behavior, 27, 419-429. doi: https://doi.org/10.1002/ab.1027

Farrington, D. P. (1993). Understanding and preventing bullying. En M. Tonry (Ed.), Crime and justice:A review of research (Vol. 17, pp. 381-458). Chicago: University of Chicago Press.

Fernández, M. (2013). Estudio de los roles en el acoso escolar: Adopción de perspectivas e integración en el aula. (Tesis doctoral). Universidad Complutense de Madrid, España. Recuperado de https://core.ac.uk/reader/19724998

Garaigordobil, M. (2010). Efectos del programa "Dando pasos hacia la paz" sobre factores cognitivos y conductuales de la violencia juvenil. Behavioral Psychology, 18(2), 277-295. Recuperado de http://www.sc.ehu.es/ptwgalam/art completo/2010/10\%20CEP-A\%20 BP\%20PC.pdf

Gimeno, J. (1976). Autoconcepto, sociabilidad y rendimiento escolar. Madrid: Ministerio de Educación y Ciencia.

Gómez, A. (2013). Bullying: El poder de la violencia. Una perspectiva cualitativa sobre acosadores y víctimas en escuelas primarias de Colima. Revista Mexicana de Investigación Educativa, 18(58), 839-870. Recuperado de http://www.scielo.org.mx/scielo.php?script=sci arttext\& pid $=$ S1405-66662013000300008

González, A. (2016). Programa TEl. Introducción. España:TEl. Recuperado de http://programatei. com/programa-tei/\#ch1

Hamodi, C. (2014). ¿Transmiten los libros de texto el valor de la igualdad desde la perspectiva de género? Estudio del lenguaje icónico de dos editoriales. REIRE, Revista d'Innovació i Recerca en Educació, 7(1), 30-55. Recuperado de http://revistes.ub.edu/index.php/REIRE/article/ view/reire2014.7.1713/12684 
doi: http://dx.doi.org/10.15359/ree.23-3.3

URL: http://www.una.ac.cr/educare

CORREO: educare@una.cr

Hamodi, C. y de Benito, Y. (Julio, 2018). Análisis del clima de aula: La sociometría. En VII Congreso Internacional Multidisciplinar de Investigación Educativa: La marcha de las ciencias de la educación (CIMIE 18). Zaragoza, España.

Hamodi, C. y Contreras, C. (2018). University training about gender equality: Approach to reality and socio-affective method as sensitizing strategies. Form@re - Open Journal per la formazione in rete, 18(2), 186-196. doi: http://dx.doi.org/10.13128/formare-23344

Hamodi, C. y Jiménez, L. (2018). Modelos de prevención del bullying: ¿Qué se puede hacer en educación infantil? IE Revista de investigación educativa de la REDIECH, 9(16), 29-50. Recuperado de http://www.rediech.org/ojs/2017/index.php/ie rie rediech/article/ view/106/152

Jares, X. R. (1995). Los sustratos teóricos de la educación para la paz. Cuadernos Bakeaz, 8, 1-12. Recuperado de http://www.aebarbiana.org/wp-content/uploads/2010/03/Los-sustratoste\%C3\%B3ricos-de-la-Educaci\%C3\%B3n-para-la-Paz.pdf

Jares, X. R. (2004). Educar para la paz en tiempos difíciles. Bilbao: Bakeaz.

Jordán Sierra, J. A., Besalú, X., Bartolomé Pina, M., Aguado Odina, T., Moreno García, C. y Sanz, M. (2004). La formación del profesorado en educación intercultural. Madrid: Catarata.

Kuz, A. y Falco, M. (2013). Herramientas sociométricas aplicadas al ambiente áulico. En Congreso Nacional de Ingeniería Informática/Sistemas de información. LINSI, Universidad Tecnológica Nacional, Facultad Regional, La Plata. Recuperado de http://conaiisi.frc.utn.edu.ar/ PDFsParaPublicar/1/schedConfs/4/93-456-1-DR.pdf

Monjas, M. I. (2000). La timidez en la infancia y en la adolescencia. Madrid: Pirámide.

Montañés, M., Bartolomé, R., Parra, M. y Montañés, J. (2009). El problema del maltrato y el acoso entre iguales en las aulas. Ensayos. Revista de la Facultad de Educación de Albacete, 24, 1-13. Recuperado de https://dialnet.unirioja.es/servlet/articulo?codigo=3282831

Morales-Ramírez, M. E., y Villalobos-Cordero, M. (2017). El impacto del bullying en el desarrollo integral y aprendizaje desde la perspectiva de los niños y niñas en edad preescolar y escolar. Revista Electrónica Educare, 21(3), 1-20. doi: https://doi.org/10.15359/ree.21-3.2

Olweus, D. (1994). Bullying at school: Basic facts and effects of a school based intervention program. Child Psychology and Psychiatry, 35(7), 1171-1190. doi: https://doi. org/10.1111/j.1469-7610.1994.tb01229.x

Olweus, D. (2013). School bullying: Development and some important challenges. Annual Review of Clinical Psychology, 9, 751-780. doi: https://doi.org/10.1146/annurevclinpsy-050212-185516 
Ortega, R. (2010). Treinta años de investigación y prevención del "bullying" y la violencia escolar. En R. Ortega (Coord.), Agresividad injustificada, bullying y violencia escolar (pp. 15-32). Madrid: Alianza Editorial.

Palomero, J. E. y Fernández, M. R. (2001). La violencia escolar: Un punto de vista global. Revista Interuniversitaria de Formación de Profesorado, 41, 19-38. Recuperado de http://w.aufop. com/aufop/uploaded files/articulos/1247352775.pdf

Pineda, I., Renero, L., Silva, Y., Casas, E., Bautista, E. y Bezanilla, J. M. (2009). Utilidad del sociograma como herramienta para el análisis de las interacciones grupales. Psicologia para América Latina, 16. Recuperado de /pepsic.bvsalud.org/scielo.php?script=sci arttext\&pid=S1870 350X2009000100009\&lng=pt\&tlng=es

Rigby, K. y Smith, P. K. (2011). Is school bullying really on the rise? Social Psychology of Education, 14(4), 441-455. Recuperado de https://link.springer.com/article/10.1007/s11218-011-9158-y

Rodríguez, A. (2001). El sociograma, una técnica útil para representar las relaciones informales. En A. Rodríguez Pérez y D. Morera Bello (Eds.), El sociograma. Estudio de las relaciones informales en las organizaciones (pp. 43-51). Madrid: Pirámide.

Ruiz de Lobera, M. (2004). Metodología para la formación en educación intercultural. Madrid: Catarata.

Smith, P. K. (2016). Bullying: Definition, types, causes, consequences and intervention. Social and Personality Psychology Compass, 10(9), 519-532. doi: https://doi.org/10.1111/spc3.12266

UNESCO. (1983). La educación para la cooperación internacional y la paz en la escuela primaria. París: Autor. Recuperado de http://unesdoc.unesco.org/images/0006/000633/063388so.pdf

Van den Berg, Y. y Gommans, R. (2017). Computer-based methods for collecting peer nomination data: Utility, practice, and empirical support. En P. E. L. Marks y A. H. N. Cillessen (Eds.), New directions in peer nomination methodology. New Directions for Child and Adolescent Development (pp. 61-73). San Francisco: John Wiley \& Sons. doi: https://doi.org/10.1002/cad.20207

Velasco, H. y Díaz, A. (1997). La lógica de la investigación etnográfica. Madrid: Trotta.

Vidal, C. y Caireta, M. (s. f). Metodología socioafectiva y aprendizaje cooperativo en la educación universitaria. Barcelona: Universitat Autònoma de Barcelona. Recuperado de http:// escolapau.uab.cat/img/programas/educacion/publicacion011e.pdf

Williford, A., Boulton, A., Noland, B., Little, T. D., Kärnä, A. y Salmivalli, C. (2012). Effects of the KiVa anti-bullying program on adolescents' depression, anxiety, and perception of peers. Journal of Abnormal Child Psychology, 40(2), 289-300. doi: https://doi.org/10.1007/s10802-011-9562-y 
doi: http://dx.doi.org/10.15359/ree.23-3.3

URL: http://www.una.ac.cr/educare

CORREO: educare@una.cr

Nombre y apellidos:

\section{Apéndice A. Test sociométrico}

Fecha: Curso:

a. ¿A qué compañeros o compañeras elegirías para trabajar en clase? ¿Por qué?

b. ¿A qué compañeros o compañeras NO elegirías para trabajar en clase? ¿Por qué?

c. ¿A cuáles de tus compañeros o compañeras invitarías a tu cumpleaños? ¿Por qué?

d. ¿A cuáles de tus compañeros o compañeras NO invitarías a tu cumpleaños? ¿Por qué?

\section{Cuestiones sobre las "percepciones sobre los roles" que cada estudiante posee en el aula:}

Quién crees que es el chico o chica de tu clase que destaca por:

a. Tener muchos amigos y amigas

b. Tener pocos amigos y amigas

c. Llevarse bien con el profesorado

d. Llevarse mal con el profesorado

e. Ser simpático o simpática con los compañeros y las compañeras

f. Ser antipático o simpática con las demás personas

g. Su capacidad para entender a las demás personas

h. No entender a las demás personas

i. Su capacidad para resolver conflictos entre compañeros y compañeras

j. Querer llamar siempre la atención 


\section{Apéndice B: Informe de evaluación}

Marca una cruz, según tu opinión, en las siguientes tablas. Estas son las equivalencias:

$1=$ nada $; 2=$ un poco; $3=$ bastante; $4=$ totalmente

Es importante que reflexiones sobre cada una de ellas y respondas lo que tú consideres.

\begin{tabular}{|c|c|c|c|c|}
\hline ÍTEM & 1 & 2 & 3 & 4 \\
\hline 1. Me he implicado y he tenido interés en la realización de las actividades. & & & & \\
\hline 2. He aprendido a comunicarme respetando las opiniones de las demás personas. & & & & \\
\hline $\begin{array}{l}\text { 3. Las actividades me han servido para reforzar mi amistad con los compañeros y las } \\
\text { compañeras. }\end{array}$ & & & & \\
\hline 4. Ahora conozco más a mis compañeros, compañeras y a mí mismo o a mí misma. & & & & \\
\hline $\begin{array}{l}\text { 5. He sido capaz de aplicar todo lo que he trabajado en clase, fuera de ella: con mis } \\
\text { amigos y amigas, en casa, en la calle, haciendo deporte... }\end{array}$ & & & & \\
\hline $\begin{array}{l}\text { 6. A partir de ahora voy a ser capaz de ponerme en el lugar del otro ser cuando tenga } \\
\text { un problema. }\end{array}$ & & & & \\
\hline 7. Si alguna vez veo un caso de bullying, voy a ser valiente. & & & & \\
\hline 8. Me gustaría seguir trabajando en este tipo de actividades. & & & & \\
\hline
\end{tabular}

